\title{
Analysis of Testicular Tissue Specific Expression of Mouse Novel Genes Dnajc5b and Cymg1
}

\author{
Xueqin Zheng, Shuai Xu, Zhen Xiang and Yang Xiang* \\ Department of Chemistry and Chemical Enginerring, Hunan Institute of Science and Technology, Yueyang, Hunan 414000, China
}

\begin{abstract}
Mouse Dnajc5b and Cymg1 genes are new genes that we cloned specifically in mouse testis. Dnajc5b is a homolog of DNAJ (HSP40), subfamily C, and the second of member 5, so named Dnajc5b, which is located on chromosome 3 of mouse and contains five exons with a total length of 947 bp. The reading frame is 597 bp and encodes a total of 199 amino acids. Dnajc5b was specifically expressed in mouse testis as shown by multi-tissue RT-PCR. Dnajc5b was weakly expressed in the first week and the second week after the mice were born, and the expression was enhanced in the third week. The greatest expression was in the eighth week of sexual maturity. At the same time, in situ hybridization results showed that Dnajc5b gene was specifically expressed in mouse spermatogonia, and its expression was positively correlated with testicular development. Cymg1 (GenBank accession No. AY600990), which has a full length of $0.78 \mathrm{~kb}$, and contains four exons and three introns, was cloned from a mouse testis cDNA library. The gene is located in the 2G3 area of chromosome 2. The full cDNA encompasses the entire open reading frame, encoding 141 amino acid residues. CYMG1 has a 44\% (48/108) identity with mouse CRES and 30\% (42/140) identity with mouse cystatin C. Northern blot analysis showed that the Cymg1 is specifically expressed in adult mouse testes. Immunohistochemistry revealed that the CYMG1 protein was expressed in mouse testes spermatogonium, spermatocytes, round spermatids, elongating spermatids and spermatozoa. RT-PCR results also showed that Cymg1 was expressed in mouse testes and spermatogonium. The Cymg1 expression level varied in different developmental stages. The Cymg1 expression level in the testes over different developmental stages correlates with the mouse spermatogenesis and sexual maturation process.
\end{abstract}

Key words: Dnajc5b, Cymg1, gene, testis, expression.

\section{Introduction}

There are approximately 2,000 genes expressed in the testis, but how the known genes interact with other genes to regulate spermatogenesis and whether other unknown genes are involved in the regulation of spermatogenesis is not fully understood. Many genes related to testicular specific expression genes and spermatogenesis have been cloned and proved to be closely related to sperm formation [1]. At the same time, the regulation of spermatogenic cell apoptosis is a complex process involving multiple factors, more new regulatory molecules and the new function of knowing molecules has yet to be discovered. Therefore, the cloning and functional identification of spermatogenic cell apoptosis-related genes is helpful

Corresponding author: Yang Xiang, Ph.D., professor, research fields: molecular and cell biology research. for understanding the regulation mechanisms of many known genes/unknown genes expressed in testicular tissues involved in spermatogenesis, also facilitating the identification of external death signals. In this study, the Dnajc5b and Cymg1 genes are new genes found for testicular-specific expression. In particular, the mouse Dnajc5b gene and the human Dnajc5b gene have a high homology of $89.5 \%$, while the Rcet1 homology is $73.3 \%$. Therefore, the study of mouse Dnajc5b and Cymg1 genes plays an important role in speculating and treating human testicular tissue cases and reproductive health. By further researching testicular development and sperm formation mechanism, as well as treating reproductive diseases and reproductive diseases, the issue of genetic reproduction has important implications [2, 3]. These will help to elucidate the molecular mechanism of human male spermatogenesis, and it is of great 
significance for exploring the etiology and pathological mechanism of male infertility [4].

Dnajc5b is a homolog of DNAJ (HSP40), subfamily $\mathrm{C}$, and the second of member 5 , so named Dnajc5b, which is located on chromosome 3 of mouse and contains five exons with a total length of $947 \mathrm{bp}$. The reading frame is $597 \mathrm{bp}$ and encodes a total of 199 amino acids. Dnajc5b was specifically expressed in mouse testis detected by multi-tissue RT-PCR. Dnajc5b was weakly expressed in the first week and the second week after the mice were born, and the expression was enhanced in the third week. The greatest expression was in the eighth week of sexual maturity. At the same time, in situ hybridization results indicated that the Dnajc5b gene was specifically expressed in mouse spermatogonia. The pEGFP-C3/Dnajc5b transfection experiment indicated that the Dnajc5b protein was expressed in the cytoplasm.

Dnajc5b study showed that Dnajc5b is specifically expressed in testis tissue, and its expression level is positively correlated with testicular developmental stage; since Dnajc5b contains Dnaj domain and HSP40 structural site, its subcellular localization in cytoplasm, Dnajc5b may have function of molecular chaperone or heat shock proteins.

By electrical hybridization, a novel gene, Cymg1 (GenBank accession No. AY600990), which has a full length of $0.78 \mathrm{~kb}$, and contains four exons and three introns, was cloned from a mouse testis cDNA library. The gene is located in the $2 \mathrm{G} 3$ area of chromosome 2 . The full cDNA encompasses the entire open reading frame, encoding 141 amino acid residues. The protein has a cysteine protease inhibitor domain that is related to the family 2 cystatins but lacks critical consensus sites important for cysteine protease inhibition. These characteristics are seen in the CRES subfamily, which are related to the family 2 cystatins and are expressed specifically in the male reproductive tract. CYMG1 has a 44\% (48/108) identity with mouse CRES and 30\% (42/140) identity with mouse cystatin C. Northern blot analysis showed that the Cymg1 is specifically expressed in adult mouse testes.

\section{Materials and Methods}

\subsection{Main Materials}

Male mice were raised in 10,000 $\mathrm{G}$ animals laminar flows (light 14 h, dark 10 h). E. coli Top10, DH-5a, BL21 and its derivative bacteria: all E. coli cell lines, used to prepare competent cells, preserved in this laboratory. pMD18-T vector, pEGFP-C3 vector, pET28b vector. Gene cloning primer: Dnajc5bF (695 bp) Gene expression primer: pEGFP-C3-Dnajc5bF. In addition, various reagent kits, spermatogonia, are required.

\subsection{Biological Information Analysis and Main Experimental Methods}

Using bioinformatics analysis, the homology of mouse Dnajc5b gene protein was compared, and the nucleic acid sequence of the gene was obtained. The EST sequence sites of mouse Dnajc5b and Rcet1 were obtained, and the obtained Dnajc5b gene sequence/protein sequence was compared. The database was compared to find the restriction sites in the mouse Dnajc5b gene, which were used to design primers, and then constructed the expression vector, and analyzed the structure of the mouse Dnajc5b protein. At the same time, RT-PCR, in situ hybridization and subcellular localization techniques were used to analyze the spatial and temporal differences and localization of Dnajc5b gene.

EST searching and gene cloning/bioinformatic analysis of the cDNA sequence of Cymg1 and protein characterization of CYMG1/RNA isolation/PCR amplification of the full length Cymg1/RT-PCR analysis/Northern blotting/Protein expression/Construction recombinant plasmid/Cell culture and transfection/Immunohistochemistry.

Cloning of Genes. Mouse testicular tissue RNA was extracted by Trizol. The mRNA of mouse testis was extracted by Trizol method as a template and amplified by RT-PCR. RT-PCR is a technique that 
combines the polymerase chain reaction of RT and cDNA. Random primers can also be used to synthesize the first strand of cDNA. Then, using the first strand of cDNA as a template and the primer of the target gene as a primer, the double-stranded cDNA of the target gene, that is, the target gene, can be obtained by PCR reaction.

Using the cDNA obtained by reverse transcription as a template, a cloning primer: Dnajc5bF 5'-GACAGAGCAGGGGAAGAACA-3'; Dnajc5bR 5'-CCACGGGCCAACATTAAGAG-3' was amplified by PCR.

High-fidelity PCR was used to clone the target gene or T-A clone, through the preparation of competent cells, transformation, screening and identification of positive monoclonal bacteria, plasmid extraction and other series of operations.

EST Searching and Gene Cloning. The DDD, BLAST [5] and ExPASy software packages were used to search for ESTs [6] in the mouse testis library, and these ESTs were electrically elongated by using the CAP Sequence Assembly Machine software. Cymg1 was cloned, which was supported by 61 ESTs, two of which came from mouse ovary and brain, the others came from mouse testis.

Protein Expression. The Cymg1 cDNA was amplified with RT-PCR from fresh mouse testis, then cloned into the pMD18-T vector and the recombinant plasmid was sequenced. Then Cymg1 cDNA was cloned into the SacI and HindIII sites of pQE30. E. coli M15 was used as host for prokaryotic expression of the pQE30/Cymg1 and subject to IPTG (isopropyl- $\beta$ - D-thiogalactopyranoside) induction.

\subsection{Dnajc5b Differential Expression Detection}

2.3.1 Differential Expression Detection of Different Tissues in Mice

Using semi-quantitative PCR technology to identify the true expression of Dnajc5b in various tissues of mice,

Dnajc5bF5'-GACAGAGCAGGGGAAGAACA-3',
Dnajc5bR5'-CCACGGGCCAACATTAAGAG-3' was used to identify the differential distribution of mRNA levels of Dnajc5b in mouse 13 tissues. At the same time, GAPDH was amplified and referenced in mouse tissues of 13. The first strand of mouse multi-tissue cDNA was used as a template.

2.3.2 Differential Expression Detection at Different Developmental Stages

In order to identify the true expression of Dnajc5b in mouse testis at different stages, testicular tissue mRNA was extracted at 1 week, 2 week, 3 week, 4 weeks, 5 week, 8 week, and 6 month, and reverse transcribed into the first strand of cDNA. Dnajc5bF5'-GACAGAGCAGGGGAAGAACA-3'Dna jc5bR5'-CCACGGGCCAACATTAAGAG-3' was set to identify the difference in mRNA levels of Dnajc5b in mouse testis tissues at different times by semi-quantitative PCR technique.

2.3.3 Detection of Differential Expression of Dnajc5b in Mouse Testis

Setting the mRNA Sequence of the Target Gene Dnajc5b. (1) 5'-TGTAA CGCAC CCAAC CAAAG ACAGC GAGCT TTGTC-3'

(2) 5'-GAACA ATTTG GAGAT GAAAA CGTTA ACACA TACTT-3'

(3) 5'-CAGAT CAGAA CTGAC ATGGA GAAAG ATATG GACTT-3'

Operating Procedures. (1) The mouse testis tissue was taken and frozen for rapid sectioning, and then $4 \%$ paraformaldehyde/0.1 M PBS (pH 7.2-7.6) containing $1 / 1000$ DFPC, fixed at room temperature for 20-30 minutes and washed thoroughly using distilled water.

(2) $\mathrm{H}_{2} \mathrm{O}_{2}$ of $30 \%$ and pure methanol were uniformly mixed, 1:50, and the slide was placed therein, and left at room temperature for 30 minutes to inactivate the endogenous enzyme, and then washed 3 times with 1/1000 DFPC-treated distilled water.

(3) Exposure of mRNA hybrid fragments (omitted)

(4) post-fixation (omitted)

(5) Pre-hybridization treatment (omitted)

(6) Probe hybridization process (omitted) 
(7) Post-hybridization treatment of probes (omitted)

(8) Adding blocking solution (omitted)

(9) Add biotin marker to rat digoxin (slightly)

(10) Add SABC (slightly)

(11) Add biotin-labeled peroxidase (omitted)

(12) DAB color development (omitted)

(13) Alcohol dehydration, xylene transparent, and sealing.

2.3.4 Subcellular Localization Technique to Detect Differential Expression of Dnajc5b

Construction of pEGFP-C3/Dnajc5b Eukaryotic Expression Vector (As shown in Fig. 1). After PCR amplification with the PEGFP-C3/Dnajc5b primer, the DNA product containing the complete open reading frame of Dnajc5b gene in the PCR product was detected by $1.5 \%$ agarose gel electrophoresis.

To prevent base mutation during PCR, high-fidelity enzymes are used for PCR to obtain right gene fragments. And $1.5 \%$ agarose gel was used for electrophoresis detection.

After digesting the high-fidelity PCR product and the corresponding pEGFP-C3 empty vector with the corresponding restriction enzymes of EcoRI and BamHI, the double-cut products were subjected to gel
Dnajc5b PCR ProductsEcoRI \& BamHI Double digestion products

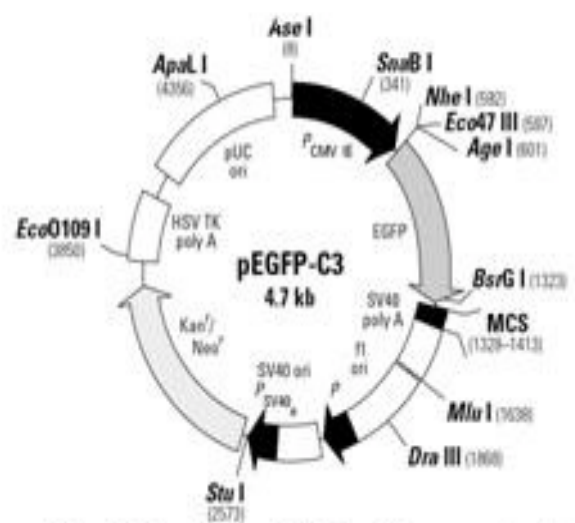

EcoRI \& BamHI Double enzyme digestion

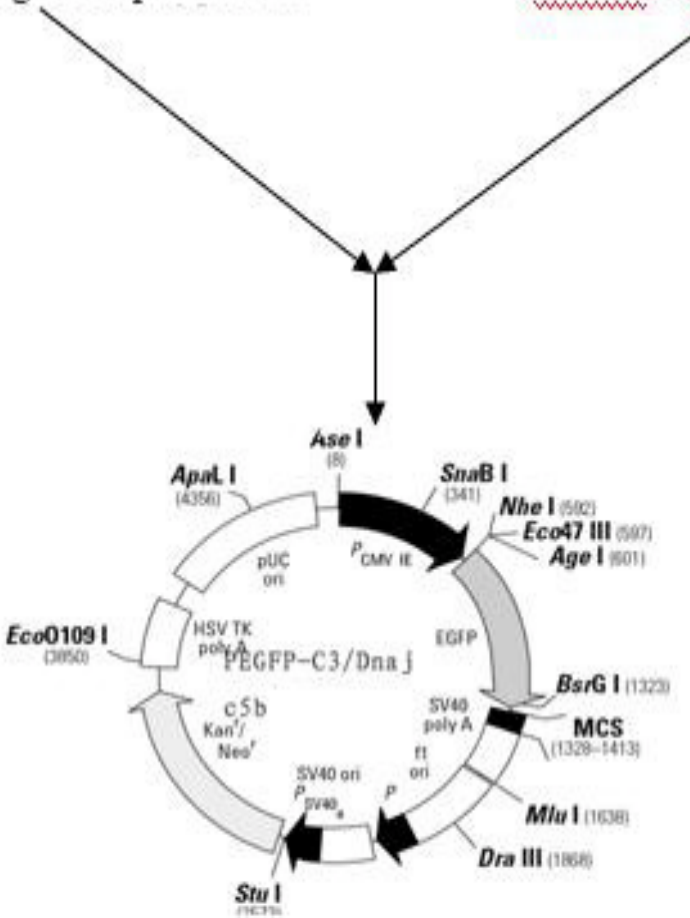

Fig. 1 Construction of recombinant eukaryotic expression vector PEGFP-C3/Dnajc5b. 
electrophoresis, and the rubber was recovered and then quantitatively linked.

The ligation product was transformed into the TOP10 competent strain, and applied to the LB-kanamycin plate. The positive bacteria were picked the next day, the transformants were identified by PCR, the plasmid was extracted, and the reading frame was verified by sequencing.

\section{Lipofectamine 2000-Mediated Gene Transfection.}

Place $96 \mu \mathrm{L}$ of serum-free medium into a sterile 1.5 $\mathrm{mL}$ EP tube into; add $3 \mu \mathrm{L}$ of Lipofectamine 2000 transfection reagent, mix gently, and leave it at room temperature for about $10 \mathrm{~min}$; then add $1 \mu \mathrm{g}$ of vector DNA (carrier DNA: Lipofectamine 2000 transfection reagent). It is $1: 3$, and the mixture is allowed to stand at room temperature for $15-30 \mathrm{~min}$. Then add $100 \mu \mathrm{L}$ of the mixture in each plate of the cell plate, and gently shake the cell plate before and after mixing, and culture the cells in a $37{ }^{\circ} \mathrm{C}, 5 \% \mathrm{CO}_{2}$ incubator. After transfection for $5 \mathrm{~h}$, the plate was washed twice with serum-containing medium, and fresh serum-containing medium (complete medium) was added, and the culture was continued for 48 hours.

Transient Transfection of pEGFP-C3/Dnajc5b Plasmid into Ovarian Cancer Cells (SKOV3). The positive control group pEGFP-C3/Dnajc5b fusion protein expression vector was set up, and the negative control group pEGFP-C3 blank vector was transfected into two groups. Six well plates were selected from 24 well plates, and a slide was placed in each group, and the cultured ovarian cancer cells (SKOV3) were inoculated to fresh serum-containing medium. By the time the cells were grown to the $90 \%$ fusion phase, the pEGFP-C3/Dnajc5b fusion protein expression vector and the pEGFP-C3 blank vector were transfected into ovarian cancer cells (SKOV3) by Lipofectamine2000-mediated transfection kit. Then, the cells were cultured in a $\mathrm{CO}_{2}$ incubator at $37{ }^{\circ} \mathrm{C}$ for 4-6 $\mathrm{h}$, and then added to the serum medium to continue the culture. The slides were taken $48 \mathrm{~h}$ after transfection, washed three times with PBS buffer, then fixed with 1\% paraformaldehyde for about 10 minutes, washed twice with PBS buffer, and then treated with $50 \%$ propanol to $50 \%$ methanol. For 30 seconds, in order to increase the permeability of the cells, the cells were washed 4 times with PBS buffer, and then the cells were stained with DAPI for 20 minutes to record the subcellular localization of the target protein by laser confocal microscopy.

\section{Results and Analysis}

\subsection{Dnajc5b Differential Expression Analysis}

\subsubsection{Differential Expression Analysis of Dnajc5b} in Different Tissues of Mice

Thirteen (13) tissues of testis, epididymis, ovary, liver, lung, large intestine, small intestine, muscle, brain, cerebellum, heart, kidney and liver of 8 weeks old adult mice were extracted and reversely transcribed into cDNA for RT-PCR detection and analysis as follows: Dnajc5bF: 5'-GACAGAGCAGGGGAAGAACA-3' Dnajc5bR: 5'-CCACGGGCCAACATTAAGAG-3'. The detection fragment size was $695 \mathrm{bp}$, and the results showed that the mouse Dnajc5b gene was specifically expressed in mouse testis, and was not expressed or weakly expressed in other tissues. At the same time, the housekeeping gene GAPDH was stably expressed in various tissues (Fig. 2). The PCR products were sequenced correctly.

3.1.2 Differential Expression Analysis of Dnajc5b in Different Developmental Stages of Mouse Testis

The testicular tissue RNA was extracted from mice at different stages of 1 week, 2 weeks, 3 weeks, 4 weeks, 5 weeks, 6 weeks, 8 weeks, and 6 months. RT-PCR was performed by reverse transcription into cDNA. The primer was Dnajc5bF: 5'. -GACAGAGCAGGGGAAGAACA-3' Dnajc5bR: 5'-CCACGGGCCAACATTAAGAG-3' detection fragment was $695 \mathrm{bp}$ in size, and the results showed that the expression of mouse Dnajc5b gene was different at different stages of mouse testis tissue. 


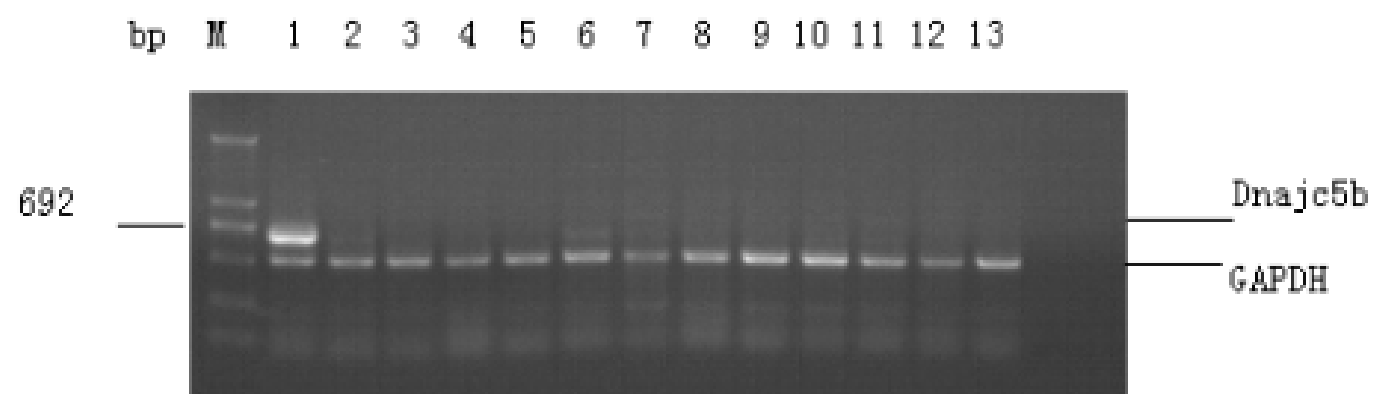

Fig. 2 Multi-tissue RT-PCR.

M: DNA Marker; 1-testis; 2-epididymis; 3-ovary; 4-liver; 5-stomach; 6-lung; 7-small intestine; 8-large intestine; 9-brain; 10—cerebell; 11-heart; 12-kidney; 13-muscle.

Dnajc5b was expressed at the 3rd week after birth. The expression was weaker before 3 weeks of birth, and the expression was gradually increased from the 3rd week after birth, and the expression was strongest at the 8th week of sexual maturation, and then weakened. Therefore, its expression is positively correlated with the testicular development period (As shown in Fig. 3).

3.1.3 Analysis of Differential Expression of Dnajc5b in Testis

Mature normal testicular tissue has different cells in the developmental stages of the seminiferous tubules. The outermost layer is spermatogonial cells, followed by spermatocytes, and then with various developmental sperm cells and mature spermatozoa. Since different developmental stages, including dividing and already differentiated cells, can be detected in same seminiferous lumen section, expression level of new gene mRNA in different developmental stages can be observed simultaneously by tissue in situ hybridization. Thus, it is possible to explore which developmental stage of the gene is expressed in the testis.

As shown in Fig. 4, it can be seen from the in situ hybridization of testicular tissue that there is a strong brown-yellow hybridization signal in Fig. 4A, and the signal is found only in the spermatogonial cells by comparison, and no signal appears in the spermatocytes. Thus, Dnajc5b is expressed in the spermatogonia of testicular tissue cells, and expression in other cells is likely to participate in the correct folding of proteins in spermatogonia to act as a molecular chaperone. This provides preliminary preparation for the subsequent study of the spermatogenesis mechanism of Dnajc5b.

In order to understand the regulation in Dnajc5b, we isolated the total RNA from different testis and the total RNA of different tissues for RT-PCR analysis, and found that Dnajc5b gene was not expressed or weakly expressed in mice for three weeks. The expression of testis development in mice gradually increased, reaching the highest in the eighth week. The RT-PCR analysis of different tissues showed that the Dnajc5b gene was specifically expressed in mouse testis but not expressed or weakly expressed in other tissues. The results of in situ hybridization assay preliminarily concluded that Dnajc5b has a signal in mouse spermatogonia, and the protein encoded by this gene may be involved in the differentiation and regulation of sperm cells. Further subcellular localization experiments showed that the Dnajc5b EGFP fusion protein showed a fluorescent signal in the cytoplasm, but did not appear in the nucleus, which was not consistent with the bioinformatics expression in the nucleus.

\subsection{Mouse Cymg1 Gene Expression Analysis}

3.2.1 RT-PCR in Mouse Multi-tissues and Different Developmental Stages

The results show that Cymg1 (512 bp) was expressed strongly in adult testes but not in other tissues. G3PDH was expressed in all kinds of tissues. It shows 


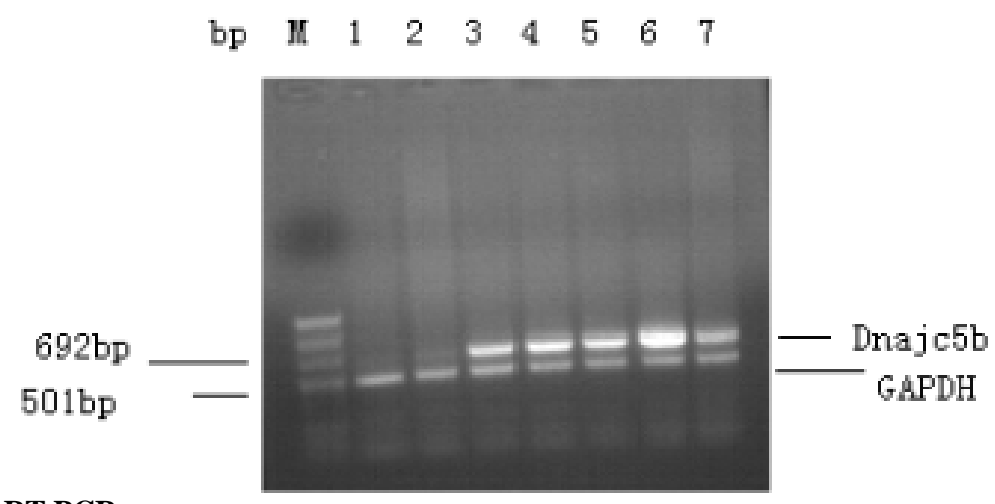

Fig. 3 Different period RT-PCR.

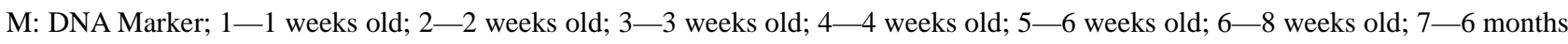
old.

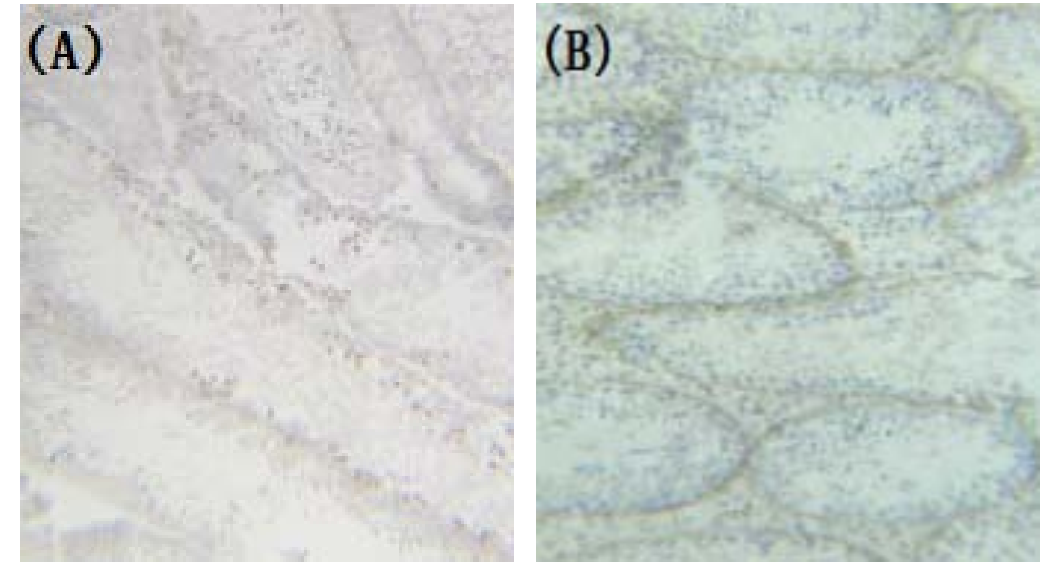

Fig. 4 In situ hybridization of Dnajc5b gene in normal adult mouse testis tissue (160X).

(A): normal adult mouse testis tissue; (B): normal adult mouse testicular tissue negative control (no Dnajc5b probe).

that Cymg1 was expressed in spermatogonial cells, and that Cymg1 was expressed in the testes of mice at different developmental stages, and that G3PDH was expressed in the testes of mice at all different development stages.

\subsubsection{Northern Blotting}

To further investigate the transcription level of Cymg1 in mouse adult multi-tissues and the transcript length, Northern blot analysis was carried out. Northern blotting showed that there was a $0.78 \mathrm{~kb}$ transcript only from adult testes. These results further confirmed the results above.

\subsubsection{Immunohistochemistry}

The results show that Cymg1 was expressed in mouse testis spermatogonium cells, spermatocytes, round spermatids, elongating spermatids and spermatozoa.

\section{Conclusion}

In this study, the specific expression of Dnajc5b gene in mouse testis tissue was detected by RT-PCR. Dnajc5b was detected in the testis tissue of mice at different stages before the three weeks of birth, and the expression was enhanced from the third week of birth. In the eighth week, the expression reached the maximum; at the same time, the expression of the gene in mouse testis was detected by in situ hybridization. The results showed that Dnajc5b gene was specifically expressed in mouse spermatogonia; and the eukaryotic expression vector of pEGFP-C3/Dnajc5b was constructed with Lipo2000 gene transfection method. The pEGFP-C3/Dnajc5b fusion vector was transfected into ovarian cancer cells (SKOV3) to obtain the Dnajc5b gene localized in the cytoplasm. 
The results showed that Dnajc5b was specifically expressed in testis tissue, and its expression was related to testicular development. Since Dnajc5b contains Dnaj domain and chaperone domain, its subcellular localization is in cytoplasm, so it is speculated that Dnajc5b may have molecular chaperone function and participate in the correct folding of proteins in spermatogonia.

In the present study, Northern blot analysis showed that Cymg1 is specifically expressed in adult mouse testes. Cell location studies showed that the GFP-tagged CYMG1 protein is localized in the cytoplasm of HeLa cells, so CYMG1 is a cytoplasm protein. Immunohistochemistry revealed that the CYMG1 protein is expressed in mouse testis spermatogonium, spermatocytes, round spermatids, elongating spermatids and spermatozoa. RT-PCR results also showed that Cymg1 is expressed in mouse testis and spermatogonium. Studies of multi-tissues and testes at different developmental stages showed that Cymg1 is expressed specifically in adult testis. These results are in accordance with the results of Northern blot analysis, which also showed that Cymg1 is specifically expressed in adult mouse testes. In addition, the RT-PCR results also showed that the Cymg1 expression level varied in testis tissue in different developmental stages: it was low 1 week postpartum, steadily increased from 2 to 5 weeks postpartum, then was highest in 7 weeks postpartum. The expression level at 5 weeks postpartum was maintained during 13 to 57 weeks postpartum. These results correlate with the mouse spermatogenesis and sexual maturation process. All these indicate that Cymg1 might play an important role in mouse spermatogenesis and sexual maturation.

Our study provides two candidate genes for testicular-specific expression of new genes, enriching the understanding of the heat shock protein family and the cysteine protease inhibitor superfamily, and further studying the mechanism of spermatogenesis between the two genes. The expression of testicular development lays the foundation.

\section{Acknowledgment}

This work was supported by Hunan Provincial Natural Science Foundation of China (No. 2017JJ2109).

\section{References}

[1] Zhang, R. X., and Nie, D. S. 2008. "Cloning and Expression Profiling of a Mouse Testis-Specific New Gene mtIQ2 Analysis.” Life Science Research 12 (2): 145-9.

[2] Mirzaei, M. R., Asadi, M. H., Mowla, S. J., et al. 2016. "Down-Regulation of HSP40 Gene Family Following OCT4B1 Suppression in Human Tumor Cell Lines.” Iranian Journal of Basic Medical Sciences 19 (2): 187-93.

[3] Li, Z. 2014. "Study on the Function and Mechanism of Human Spermatogenesis Related Gene DNAJB13 in Sperm Motility.” Central South University.

[4] Li, W. N. 2007. "Preliminary Study on the Relationship between TSARG6 and AR in Human Spermatogenic Cell Apoptosis-Related Genes.” Central South University.

[5] Stephen, F. M., Barry, O. D., Simon, J. F., Hugh, R. B., Guenole, S., and Peter, P. D. 2003. "Digital Extractor: Analysis of Digital Differential Display Output.” Bioinformatics 19 (12): 1594-5.

[6] Qian, J., Zhang, X. M., Li, X. L., Wang, J. R., Li, W. F., Wang, R., and Li, G. Y. 2002. "Identification of Digital Differential Expression Patterns of a Novel Human Gene (UBAP1) by an Expressed Sequence Tag Strategy." Ai Zheng 21 (3): 225-8. 